\title{
Archaeologia
}

http://journals.cambridge.org/ACH

Additional services for Archaeologia:

Email alerts: $\underline{\text { Click here }}$

Subscriptions: Click here

Commercial reprints: $\underline{\text { Click here }}$

Terms of use : $\underline{\text { Click here }}$

\section{XVIII.-Benefactions of Thomas Heywood, Dean (1457-1492), to the Cathedral Church of Lichfield}

\section{J. Charles Cox}

Archaeologia / Volume 52 / Issue 02 / January 1890, pp 617 - 646

DOI: 10.1017/S0261340900007633, Published online: 25 January 2012

Link to this article: http://journals.cambridge.org/abstract S0261340900007633

How to cite this article:

J. Charles Cox (1890). XVIII.-Benefactions of Thomas Heywood, Dean (1457-1492), to the Cathedral Church of Lichfield. Archaeologia, 52, pp 617-646 doi:10.1017/S0261340900007633

Request Permissions : $\underline{\text { Click here }}$ 


\section{XVIII.-Benefactions of Thomas Heywood, Dean (1457-1492), to the Cathedral Church of Lichfield. By the Rev. J. Charues Cox, LL.D., F.S.A.}

Read May 9, 1889.

The roll of the Deans of Lichfield will favourably compare with those of any of our cathedral churches of the old foundation. Several of them have been distinguished scholars and theologians; one or two of them made some mark in the history of their country at a time when high ecclesiastics were frequently statesmen; twelve of their number obtained preferment to sees episcopal and archiepiscopal; whilst three, including the present Dean (whose name will ever be associated with the noble restoration of the west front of the cathedral church), were for many years Prolocutors to the Province of Canterbury.

But there is one name connected with Lichfield cathedral church, Thomas Fisher, or, as he was more usually called from his birthplace, Thomas Heywood, which, though passed over in local or diocesan histories in a few words, is well worthy of more extended notice. For length of service and for bountiful beneficence Thomas Heywood is without a rival among the Lichfield deans. Born in 1409, he was collated to a prebendal stall in Lichfield cathedral church in 1433, so that for sixty years he was connected with the minster, for the last thirty-five of which he held the deanery. The recently arranged muniments of the Dean and Chapter of Lichfield, in conjunction with the episcopal registers and the chapter act books, enable the inquirer into the events and administration of this long and active ecclesiastical life to glean many particulars of church and decanal work, as well as of city life, during a period that has been far less enlightened by historical writings than several eras of a more remote date.

Much can be learnt of the early life of Thomas Heywood, and of the other preferments that he held; the fullest details exist of his remarkable and touching VOI. IIIT. 
election to the Deanery per inspirationem, and of the ceremonies and technicalities therewith connected; and not only is there evidence of the vigour of his ecclesiastical discipline and control over the cathedral church and its extensive peculiars, by visitations and general supervision, but ample information can be obtained of the remarkable biennial visitations that he held of the city of Lichfield, when specially appointed wardens, two for each street of importance or ward, presented at his court all manner of offenders against good morals, showing that the dean had to adjudicate in cases of incontinence and drunkenness, of late hours, of gambling, of evil language, of domestic quarrels, of neglect to extinguish fire, and of breaking the Sunday and holy days.

At the same court, too, the laity presented the clergy; for the wardens presented city priests on one occasion for saying mass inaudibly, and on another for leaving out certain words.

But all that I propose to do in this paper is to call attention to the variety and extent of his gifts to the cathedral church of Lichfield and its surroundings, and I trust that the nature of some of the gifts and the terms or language in which they are expressed may be of sufficient interest to warrant my bringing the matter before the Society, trusting that it may be, at all events, of some little interest to ecclesiologists.

In the library of the Dean and Chapter, situated over the chapter-house, and not among the muniments on the opposite side of the church, are three small folio volumes of parchment. They are bound, and erroneously lettered on the back Cantaria S. Blasii, from the first entry; their better title would be "The Benefactions of Dean Heywood."

The following is an analysis of the contents of these three small volumes:

The first volume consists of forty-five folios of rather poor parchment, twelve inches by eight. Some of the pages are a good deal soiled and marked with damp, but they are fairly written and legible throughout. The first folio is headed with the words "Cantaria Scci Blasii." The following is a summary of the contents :

f. 1. I. Decree of John Fox, chancellor of the diocese, concerning the payment of a pension of nine marks by the abbey of Hales Owen (in Shropshire) to the chantry of St. Blase, in Lichfield cathedral church. n. d.

II. Instructions for the obit of Dean Heywood's parents whilst he lived, and the same after his death. 14 August, 1456.

f. 2. III. Indenture from the abbey of Hales Owen concerning an annual rent of $£ 6$ to be by them paid for the support of the chaplain of the chantry of St. Blase, 14 April, 1466 . 
f. 3b. IV. Concession from the Dean and Chapter of Lichfield of the above grant, etc. 20 November, 1467.

f. 4b. V. Admission and reception of the warden and chaplain of the New College within the Close, and of St. Blase's chantry, etc. 22 November, 1467.

f. 6. VI. Official subscription of the foregoing by two public notaries, Thomas Colt and Thomas Reynolds. 9 February, 1467. In the Consistory Court.

f. 7. VII. Confirmation of number I. by the abbey of Welbeck, as the father abbey of Hales Owen. 3 April, 1468.

f. 9 . VIII. Ordination by Dean Heywood of the chantry of St. Blase. 16 April, 1468.

f. 12b. IX. Obit for Dean Heywood after his deatl. 9 November, 1471.

f. 14. X. Indenture between the abbey of Lilleshull, and the cathedral church of Lichfield respecting the annual payment of ten marks for the support of the chantry of Jesus and St. Anne in the latter. 4 July, 1468.

f. 16. XI. Confirmation of the above by the Bishop. 14 November, 1468.

f. 18b. XII. Ordination by Dean Heywood of the chantry of Jesus and St. Anne. 3 December, 1468.

f. 23 b. XIII. Confirmation of the above by the Bishop. 7 January 1468-9.

f. 24. XIV. Confirmation of the above, by the Chapter, and formal concession of number X. 23 December, 1468.

f. $26 \mathrm{~b}$. XV. Admission of the chantry priest of Jesus and St. Anne, by the warden, into the New College within the Close. 23 December, 1468.

f. 28. XVI. Arrangement with the sub-chanter and viears about the masses and antiphonal singing at the last-named chantry altar in the north transept. 20 February, 1472.

f. 30b. XVII. Ordination by the Dean to the same effect as the last, and also with respect to ringing the bell for those services. 9 April, 1473 .

f. 33. XVIII. Indenture between the abbey of Dale (in Derbyshire) and the chapter of Lichfield, respecting four marks annually from the former, for the use of the six vicars singing the mass of the Name of Jesus onWednesdays. March, 1471.

f. 36b. XIX. Further particulars relative to the last. 20 February, 1472.

f. 37. XX. Confirmation of number XVIII. by the abbey of Newhouse (in Lincolushire) as father abbey of Dale. 11 August, 1474.

f. 40b. XXI. Concession from the abbey of Dale as to keeping the obit of Dean Heywood within that monastery; with subscription of public notary. n. d.

f. 44. XXII. Confirmation of the last by the abbey of Newhousc. (last part missing).

f. 45. An outer leaf, embellished with various pen-trials.

The second volume consists of thirty-seven folios of parchment. The first twenty folios are carefully written, with red titles and well worked initial letters of the same colour. The capital letters throughout are also just touched with red. These folios are about twelve inches by eight, but the remainder of the 
book has smaller leaves and is written in a bearififully clear hand by a different and more accomplished scribe.

Folios 1 to 10 contain the official decree of enrolment of the two chantries, etc., founded by Dean Heywood, in the Lichfield consistory court by Thomas Reynold, vicar-general or chancellor of the diocese, wherein are recited at length:

(1). Bull of Pope Sixtus IV. pridie kl. Octobris, 1479, confirming the two chantries.

(2). Bull of Pope Sixtus IV. pridie kl. Februarii, 1481, granting indulgences to penitents visiting the chantry of Jesus and St. Anne. 7 years and 40 days.

(3). Letter from the College of Cardinals approving the indulgences. March 5, 1482.

(4). A similar letter from the Cardinals, dated March 6th, 1481.

(5). Letter approving the indulgences from Thomas, archbishop of Canterbury, and seventeen of his suffragans.

(6). Official witnessing and subscription of the above by Thomas Godsalve, notary public and chapter clerk.

(7). Official witnessing and subscription of the above by John Bagnald, notary public.

Folios 11 and 12 are blank.

Folios 13 to 15 contain a copy of a judgment in the King's Bench respecting the annual pension due to the chapter from the abbey of Lilleshull. 20 May, 22 Edward IV.

Folios 16 to 20 are blank.

On folio $20 \mathrm{~b}$ is a list of Dean Heywood's benefactions to the Lichfield chapels of SS. Mary, Michael, and Chad.

Folios 21 to 37 contain the general summary of all the benefactions of Heywood to the cathedral church. They are given in full in the Appendix to this paper.

The third volume has 63 folios. Of these, folius 1 to 55 are an exact copy of vol. i. Folio 56 is blank.

Folios 57 to 62 contain benefactions of Thomas Heywood to the vicars. These are almost identical with the latter part of vol. ii., and are written by the same scribe.

Folio 63 is blank.

The three city churches of Lichfield were, up to the time of Elizabeth, considered chapels of the minster (as well as various small churches within an area of several miles from Lichfield), and were served by priests termed the city prebendaries. Each of the three old city churches received benefactions from Dean Heywood. To the chapel of the Blessed Mary in the market place he gave the 
glazing of the principal window at a cost of $£ 8$; to Richard Ruge, carpenter, for erecting the "Rodelofte" at the entrance of the choir, $£ 1613 s$. 4d.; for erecting pillars in the nave of the chapel, $£ 21$; to Gwilerm the carpenter, and his son, for erecting a new roodloft on the north side of the said chapel, $£ 156 s .8 d$.; a gift of $£ 513 s$. $4 d$. to the gild of the chapel; and to the repair of the chests, $£ 70 s .8 d$. To the wardens of the church of St. Michael he gave $£ 10$ for fabric repairs; and 10 marks for a like purpose to the wardens of the chapel of St. Chad at Stow.

It may be of interest to give the original record of these gifts :

Data et sumptus venerabilis Magistri Thome Heywode Decani ecclesie cathedralis Lich. fact̃ infra Capella beate Marie in foro Lich. situata.

In primis solvit pro vitriacione principalis fenestre

Item solvit Ricardo Ruge Carpentario pro fabricacione de le Rodelofte in

Introitu chori ibidem

Item solvit per manus Dominorum Willelmi Hukyns et Thome Parker

pro fabricacione Columpnarum in navi Capelle

Item solvit Gwilermo Carpentario et filio ejus pro fabricacione nove

Rodelofte in parte Boriali Capelle predicte

viij ti.

xvj ti. xiij s. iiij d.

xxj łi.

xv ti. vj s. viij d.

Item cledit ad emendas possessiones Gilde ibidem applicandas et ampliandas v ti. xiij s. iiij d.

Summa totalis

Et ultra predicta dictus Magister Thomas Decanus dedit redintigracioni lxvj ti. xiij s. iiij d.

(sic) Cistarum Magistrorum Johamnis Harewode et Georgij Radelyffe vii ti. viij d.

Item dedit Gardianis ecelesie sancti Michaelis Lich' et ad fabricam ejusdem

Capelle

$x$ ti.

Item dedit Gardianis Capelle de Stow ad fabricam ejusdem

$x$ marcas.

Capelle beate Marie in foro

Licfi.

Ecclesic saneti Michaelis Lich.

Capelle de Stow.

In founding the chantry of St. Blase, its site is specially named as being opposite the episcopal seat.

He gave to this chantry all that was seemly and convenient (congruentem) for the celebration of mass there; one good mass-book with the capital letters gilt, one silver-gilt chalice of the weight of 20 ounces, and three sets of vestments being specially named. He also gave a reredos or table of alabaster to be placed at the back of the altar, carved with events in the life of Bishop Blase.

To the abbot and convent of Hales Owen ${ }^{2}$ the Dean gave two silver basins with gilt eagles (egles) in the bottom, of the weight of 69 ounces., and of the value

a An abbey of White Canons in Shropshire. 
of $£ 10$ and upwards. These silver basins, which I suppose were for the washing of the hands during mass, were to be placed on the altar of St. Kenelm the martyr in his chapel on solemn days and festivals ad Dei laudem et complacenciam et dicti martiris honorem.

In 1468 the Dean made additions to the New College of the vicars, by building a new bakehouse and brewhouse de duo bayes with two chambers over for the storing of corn and barley; he also caused the building to be re-roofed with tiles, and erected an oven and furnace at the cost of 20 marks, for the safety of his soul and for the convenience of the chaplains. He also gave to the chaplains $40 \mathrm{~s}$. for the fabric of the place where the ciphorium is situated and for the glazing of the windows, which place is then described as competens ornatus et satis honestus. The Dean also spent $20 s$. on an iron chimney (caminum ferreum) for use in the common hall of the chaplains during winter. He further gave them for the high table of the hall a good table-cloth de panno bilicino, anglice Iwolly, at a cost of $6 s .8 d$.

The chantry chapel of Jesus and St. Anne, founded by the Dean in 1468, is described as being in solario ad finem Borialem pulpitu, and in another place as erected in sublime in the north transept. Here was a glorious image of Jesus and an image of St. Anne. A cursal mass was to be said daily, and a specially solemn mass, with the best voices of the choir and with organ accompaniment, at seven o'clock every Wednesday.

To that altar he gave a mass-book, a good silver-gilt chalice and paten weighing upwards of 21 ounces, a red vestment with apparels of crimson velvet, another vestment with its apparels of blue silk, and a third vestment of black cum verbo Jhu. in pluribus partilus et vicibns conscriptum for requiem mass, also albes, and apparels, and everything that was necessary and convenient for these vestments; also two corporals and their cases, and four linen clothes for the top of the altar, and a linen cloth painted with the image of the Blessed Mary in the midst for covering over the altar after the celebration of mass. Also four ornamenta for the altar, namely, one of blue silk and three de steynet Warke; two towels, one basin "de Masselyn"; two pewter cruets; one little pax; and a cushion upon which the mass-book lies.

In 1481 the Dean gave to the same altar a vestment of white velvet, with albes, apparels, and everything pertaining, and with the name of Jesus frequently embroidered on the apparels, of the value of 5 marks.

At a later date the Dean gave to the same altar of Jesus and St. Anne, for use at the daily cursal mass, a good silver-gilt chalice and paten weighing 20 ounces, 
enamelled on the foot with an image of the Crucifix; also three vestments, one of green bawdekyn for ferial days, another of green silk embroidered with gilt stars, and a third of blue "wolstyd" with red orphreys behind and before; also a good mass-book magne quantitatis with a cover of leather; and a red book of commemorations and of the mass of Jesus and of Requiem to be celebrated every Wednesday.

In 1474, the Dean caused to be built a new chamber with a chapel over the gateway at the entrance to the vicars' houses; the chamber (et le drawth) being so arranged that the infirm vicars could hear mass in the chapel.

The Dean also gave three murras, anglice Masers, with gilt bands, of the value of $53 s$. 4 4 ., to the use of the vicars, and a good table cloth "de twyll" for the high table in their hall.

In 1477 , Dean Heywode gave a very great bell to the cathedral church, which was cast in London and cost $£ 100$. It was called "in vulgari, Ihic belle," and hung in the south tower of the west front. The bell was consecrated and hallowed on November 14th, by Robert, bishop of Achonry (in Ireland), suffragan of John, bishop of Coventry and Lichfield. The ceremonial is described with much detail, (see Appendix), and at the conclusion of it, all who had sung or taken any part therein dined joyfully with Thomas Heywood in the deanery.

In 1481 the Dean gave to the cathedral church duas capsellas, anglice monstrance, of silver-gilt, curiously wrought, for containing relics, and assigned them to the custody of Dan William Hukyns, chaplain of St. Blase's chantry and keeper of St. Chad's Head; the value of the same being $£ 413 s$.

In the following year he gave to the church, at a cost of $£ 263 s$. $4 d$., a new pair of organs magne quantitatis et forme decentis, to stand in the pulpitum or loft over the screen at the entrance of the quire. He also gave a new pair of organs, at a cost of $£ 12$, to stand in the new fabric opposite the Jesus altar, to serve for the solemn Jesus mass every Wednesday.

There are also recorded further gifts in the same year (1482) of a best altarcloth of twill for the high altar, a good altar-cloth of the same stuff for the altar of Jesus and St. Anne, another for the altar of St. Blase, and a fourth to the altar of St. Chad, "in the chapel where his head is wonderfully honoured."

The Dean, at his own proper cost, also caused all the windows of the chapterhouse to be glazed, with images of the Apostles and other pictures on the roof and walls. He also added a wooden wainscot and glazed the window in the vestibule or entry. The cost of the whole was $£ 46$. 
The cathedral church as well as the city of Lichfield seem to have prospered in a marked degree under the active as well as munificent rule of Dean Heywood. His generosity kindled the generosity of others.

The fully detailed account of the election of Dean Heywood, per inspirationem, in 1457, after describing his repeated refusals of the office, gives these as the words of his eventual acceptance :

"In the name of God, Amen. I, Thomas Heywcod, canon of the cathedral church of Lichfield, elected to the deanery of the same cathedral church by my brother canons, with regard to my consent to the said election, being urged thereto by the repeated and reiterated entreaties of my brethren, and being unwilling to resist the Divine Will, in the name of Christ whom I have invoked to my aid, and trusting in the Divine Clemency, I give my consent, to the praise and honour of God, and of His mother Mary, and of St. Chad the confessor, and of all the saints of God."

The brother canon, who acted as chronicler of the election, concludes by describing his new chief as by birth and age, as well as in morals, life, and learning, pre-eminently suited for the office, and as one who would be sure to uphold the ancient rights of their church, both in matters spiritual and temporal.

At the death of Thomas Heywood in 1492, after a decanal experience of thirtyfive years, Lichfield must have felt that the aged Dean had abundantly fulfilled the pious expectations that were entertained at his entering upon the office.

I desire to express my obligation to the Dean and Chapter of Lichfield, and particularly to Canon Lonsdale, Honorary Librarian, for entrusting to my care the three volumes described in this paper.

I have also to thank Mr. W. H. St. John Hope for transcribing for me the interesting part of Vol. II. which forms the Appendix to this paper. 


\section{APPENDIX.}

\section{Ordinacio sive fundacio Cantarie Sancti Blasii.}

In Dei nomine. Amen. Universis Christi fidelibus imnotescat. quod venerabilis vir magister Thomas Heywode. in utroque jure Bacallarius. ac in decretis doctor licentiatus. Decanus ecclesie Cathedralis Lici.i. Anno domini millesimo [quadringesimo] sexagesimo sexto. ad honorem et laudem sancte Trinitatis. sancti Cedde presulis. sancti Blasii martiris. Autoritate et consensu Reverendi in Christo patris et domini Domini Johannis Hals. Dei gracia Coventr' et Lich. episcopi. Ac eciam unanimi consensu et assensu ejusdem ecclesie Capituli. fundavit et ordinavit unam Cantariam Sancti Blasii martiris. quasi ex opposito sedis Episcopalis situatam. et unum presbiterum ad altare ejusdem Cantarie missam perpetuo celebraturum. Primo dominum Willelmum Hukyns. capellanum perpetuum ejusdem Cantarie nominavit et providit Qui capellanus et successores sui pro tempore existentes dicte Cantarie capellani. et quilibet capellanus pro tempore suo de Abbate et Conventu de Halesowen ordinis premonstratensis Wygorn' dioces' percipiet annuatim imperpetuum. Ad ffesta Michaelis et Pasche. per equales porciones Centum solidos. de Novem marcis. quas per suas literas patentes et indentatas. sigillo eorum communi sigillatas. decano et capitulo Lich. singulis annis terminis predictis solvere obligantur. Et reliquos viginti solidos de sex libris predictis capellani cantariarum et novi Collegii infra clausum Lichfielđ situatum. annụatim in terminis predictis realiter percipient. do prefatis Abbate et conventu de Halesowen. pro recepcione capellanorum dicte cantarie in ipsorum consorcium. Ac eciam quod intersint et quilibet eorum intersit in anniversariis parentum suorum dum vixerit. Et post obitum suum. In anniversariis ejusdem Magistri Thome decani. in choro et in habitu chorali. dicentes et psallentes pro anima sua et parentum suorum. et omnium in ordinacione sua nominatorum. una cum vicariis choralibus ecclesie cathedralis Lich. juxta formam ordinacionis prefati Magistri Thome Heywode decani. et concessionis dictorum capellanorum confectam. et sigillis eorum sigillatam. una cum sigillis dicti Reverendi patris. Ac decani et capituli ecclesie cathedralis Lich. sigillatam ut plenius in eisclem continetur.

Quantum repeperunt (sic) Abbas et conventus pro pencione.

Et memorandum quod de premissis sex libris annuatim solvendis per dictos Abbatem et Conventum de Halesowen et enrum successores imperpetuum decano et capitulo ecclesie cathedralis Lich. et eorum successoribus ad usus supradictos. et pro obitu suo annuatim per eosdem in Monasterio suo predicto celebrando juxta formam in dictis literis indentatis, et eorum sigillis sigillatis plenius expressatam. predictus Magister Thomas Heywode decanus supradictus solvit dompno Johanni adtune abbati. et ejusdem loci conventui quadringentas marcas legalis monete anglic.

VOL. LII. 
ad emenđ sibi possessiones. aut redditus. ex quibus possit annuatim dicta pecunia provenire. ct residuum in commodum Monasterii sive domus predicte utilitatem conversi. Tा Et prefati Abbas et Conventus cito post cum parte dicte pecunie emerunt de Bevyce Hampton possessiones et redditus suos in Civitate Lich. ac terras et pasturas suas juxta Lich. vocatas Pownes feledes. ad valorem annuum quasi viginti marcarum. licencia domini Regis ad hoc habita et concessa et mortizata in forma juris regni Anglie. If Et cum altera predicte summe porcione predicti Abbas et conventus de Halesowen quendam prioratum in diocese Wygornie ad annualem valorem viginti mareartm. nomine Todforde. suo monasterio inperpetuum ajpropriarunt.

\section{De duobus pelvis (sic) argenti.}

Insuper predictus Magister Thomas Heywode Decanus dedit ex sua devocione mera et deliberavit eisdem Abbati et Conventui duas pelves de argento, cum Egles deauratis in fundo ponderis. sexaginta novem unciarum precii decem librarum. et ultra. ut deserviant ponantur et situantur super altate $(\mathrm{sic}$ ) sancti Kenelmi martiris in capella ejusdem diebus solemnibus et festivis. ad Dei landem et complacenciam. et dicti martiris honorem.

\section{Quantum solvitur voluntarie per aldate [m].}

Et quia prefatus Beves Hampton postquam sua terras pasturas et tenementa infra Lich. et juxta ut prefertur. predictis Abbati et Conventui vendidit et deliberavit. ex quadam convencione inter eosdem facta, quandam summam annualem decem marcarum de eisdem Abbate et conventu durante vita sua habere debuit. prefatus decanus ex sua benevolencia et voluntate propria prefitos abbatem et conventum de annuali pensione novem marearum ecclesie Lich. solvenda per spacium duodecim annorum quibus prefatus Beres supervixit. pro eisdem annuatim ecclesie Lich. solvit et satisfecit.

Item tempore quodam quo prefatus decanus fuit aput (sic) Halesowen, dedit abbati ct conventui decem marcas et servientibus prout decuit.

\section{De Ornamentis capelle.}

Item idem Magister Thomas Decanus dedit et contulit omnem ornatum ad dictam cantariam sancti Blasii congruentem. Et ad celebracionem missarum ibidem per dictum dominum Willelmum Hukyns dicte cantarie capellanum modernum et successores suos imperpetuum unum bonum missale. cum literis capitalibus aureis. et unum calicem deauratum ponderantem viginti uncias tria vestimenta sacerdotalia. et alia ornamenta pro altari et apparatu ejusdem congrua et conveniencia. 
De edificio fucto in noro collegio.

Item idem Magister Thomas cito post in vita sua videlicet Anno domini Millesimo CCCC ${ }^{\text {mo }}$ sexagesimo octavo. edificari fecit suis expensis novum pistrinum et pandoxatorium de ij bayes infra domum. cum duabus cameris supra pro frumento et brasio custodiendis. Et eandem domum cantariarum eum tegulis cooperiri fecit. Ac furnum et fornacem ibidem stabilari fecit. suis propriis expensis. ad summam viginti marcarum. ad salutem anime sue. et commodum dictoruni capellanorum.

Item contulit cisdem capellanis xl. solidos. ad fabricam loci ubi ciphorium situatur. et ad vitriacionem fenestrarum ibidem. qui locus est competens ornatus. et satis honestus.

Item idem Magister Thomas Decanus predictus contulit eisdem Capellanis caminum ferreum. ad usum eorundem tempore iemali in aula eorum communi. precii viginti solidorum.

Item dedit commitive eapellanorum predictorum unum mensale bonum de panno bilicino. anglice twyly. precii vjs. viij. d. ,

\section{Ordinacio sire fundacio Cantarie Jesu et sancte Inne.}

Ad honorem nominis domini nostri Jesu Cliristi. et sancte Amno matris beatissime Marie virginis. Magister Thomas Heywod. in utroque jure Bacallarius. et decretorum doctor admissus. Ac ecclesie Cathedralis Lich. Decanus. Anno Domini Millesimo CCCC sexagesimo octavo fundavit et construxit suis expensis Cantariam perpetuum desuper fabricatam. ex parte boriali ecclesie cathedrali predicte. cum gloriosa Imagine Jesu Christi unum ibidem stantis. et cum Inagine sancte Anne ibidem eciam defixa. Et ordinavit et providit dominum Willelmum Brodbent primum capellanum perpetuum ibilem celebraturum tempore suo. Et alios capellanos successores suos ibidem perpetuo celebraturos. juxta vim et formam ordinacionis sue. auctoritate et consensu Reverendi in Christo patris et domini. Domini Johannis Hals Corentrensis et Licti. episcopi. Ac cciam unanimi consensu et assensu tocius ejusdem ecclesie cathedralis Lich. capituli ut in literis inde confectis eorum sigillis sigillatis plenius continetur. Dedat' sigilla (sic) communis pontificalis et sigilli communis capitularis in ecclesia cathedralis Lich. in loco capitulari ejusdem Septimo die mensis Januarij. Anno Domini. Millesimo. CCCC ${ }^{\mathrm{m}}$ sexagesimo octayo. T Qui quidem eapellanus et quilibet successorum suorum pro tempore suo percipict annuatim pro salario ct sustentacione sua ad duos anni terminos videlicet Invencionis Sancte Crucis. et sancti Martini in yeme per equales porciones Centum solidos de Abbate et conventu de Lylleshuff. It Et capellanus quicunque ipsius cantarie pro tempore existens. residebit in camera sibi limitatá. cum capellanis cantariarum in novo collegio eorundem. T Qui capellani sen cetus capellanorum percipient cciam de prefatis Abbate et Conrentu annuatim ad terminos predictos triginta tres solidos. et quatuor denarios de decem marcis quas Albas et Conventus de Lylleshuft tonentur annuation solvere. decano et capitulo ecclesie cathedralis Lick. prout in literis indentatis inter dictos abbatem 4 м 2 
et conventum et decanu et capitulum ecclesie Lich. inde confectis liquet manifeste. I Sed cum tresdecim solidis et quatuor denariis de predictis xxxiij ${ }^{\text {bus }}$ solidis et quatuor denariis per eos ut predicitur percipiendis predicti capellani seu cetus capellanorum collegij novi predicti. solvent decano et canonicis residenciariis et alijs ministris in obitu et exequiis seu anniversariis ipsius decani existentis omni anno. juxta formam et modum limitatum et conseriptum in literis indentatis. et inter predictos Magistrum Thomam Heywod. decanum et capitulum Lich. ac ipsos capellanos cantariarum. et prepositum et cetum eorundem factis et eorum sigillis alternatim sigillatis.

\section{Quantum dedit Abbati de Lylleshull' pro pensione.}

Et memorandum quod pro premissis decem marcis per Abbatem et conventum de Lylleshutt annuatim solvendis in terminis sancti Martini et Invencionis Sancte Crucis per equales porciones per dictos Abbatem et conventum de Lylleshuff. et eorum successores imperpetuum. Decano et capitulo ecclesie Lycћ. et eorum successoribus ad usus supradictos. Ac pro obitu suo annuatim per eosdem in monasterio suo predicto celebrando. et missam cotidianam per unum de canonicis ejusdem et septimanatim celebrandam juxta vim et formam in dictis literis indentatis inter dictos Robertum tunc abbatem et conventum de LylleshuH et eorum successores et Decanum et capitulum Lycłi. et corum successores factis. et eorum sigillis sigillatis. Ac per Reverendum patrem et dominum. dominum Johannem Coventrensis et Lich. episcopum confirmatis. et ejus sigillo sigillatis plenius expressatis. et plenius in eisdem continetur prefatus Magister 'Thomas Heywode decanus supradictus solvit dompno Roberto ad tune Abbati et conrentui ejusdem loci. quadringentas marcas legalis monete Anglie ad emendas sibi possessiones et redditus. ex quibus possit annuatim dicta pecunia provenire. Et residuum in commodum Monasterij sive domus predicte utilitatem converti. T Et prefati Robertus Abbas et Conventus cito post emerunt terras redditus et possessiones apud Brugenorthe. ac eciam hospitale sancti Johannis ibidem sibi dari et appropriari auctoritate domini regis. ac domini comitis Salopiae patroni pretensi. et omnium quorum interest consensu. plecarie sibi assignari procurarunt. ad valorem annum quadraginta marcarum. I Insuper prefatus Magister Thomas Heywode decanus dedit dicto domino Roberto ad tunc abbati ex sua mera devocione ad usum magnatum ibidem hospitancium rubiam penilenciam mius lecti de Redsay et Wolstyde. cum le curtence et dowbull' frengeth'. et cum coopertorio lecti de eadem secta valoris vij librarum. If Item fieri fecit parvas cameras in dormitorio ibidem pro quolibet canonico. Et coopertorium dormitorij. et fenestras vitreas ibidem. ad summam expensarum. T Item et contulit eisdem ad ornatum altaris capelie beate Marie unum pannum pictum cum rosis. satis congruum. cum cluobus curtence ejusdem secte precij xxxiij.s. iiij. d. Necnon temporibus. quibus illuc progrediebatur. distribuit pecunias et ministris largiter prout decuit. T Item prefatus. Magister Thomas Heywode decanus dedit prefati abbati xx ${ }^{\circ}$. die sem-

1480. tembris (sic) Anno Domini $\mathrm{M}^{\circ}$. $\mathrm{CCCC}^{\circ} \mathrm{Kxx}^{0}$ unam peciam deauratam stantem cum coopertorio. cum signo campane in capite conpertorij. ad deserviendum sibi et successoribus suis imperpetuum.

Item memorandum quod Magister Thomas Heywode decanus supradictis contulit dicto 
altari Jesu et sancte Anne in capella predicta in ecclesia cathedrali Licǩ. et capellanis perpetuis ibidem successive deservituris. et missus celebrantibus unum missale competens. calicem bonum cum patena deaurata ponderis xxj. unciarum et ultra. Et tria vestimenta sacerdotalia videlicet. unum bonum vestimentum. cum suis apparuris Rubium de crýmsýn veluet. Secundum vestimentum cum suis apparuris de blodio serico. Tertium vestimentum. de nigro. pro missa de Requiem. diebus veneris. cum nota de cantanda cum verbo Jћu. in pluribus partibus et vicibus conscriptum enm albis et paruris. et singulis alijs dictis vestimentis necessarijs et convenientibus. cum cluobus corporalibus cum duabus tecis pro eisdem. Necnon quatuor lintheamina honesta et decencia super idem altare. ac lintheamen depictum. cum Innagine beate Marie in medio. ad cooperiendum ipsum altare post complecionem missarum ibidem celebratarum. Item quatuor ornamenta pro altari videlicet unum de blodio serico. et tria de steynet Warke. Item duo manutergia. Item $\mathbf{j}$ pelvis de Masselyn. Item dne fiole stannee. Item $\mathbf{j}$ paxillum. Item $\mathbf{j}$ pulvinar super quo jacet missale. Tf Pro quibus libro. calice. vestimentis. et indumentis altaris calice. ac singulis supradictis. sentencia excommunicacionis lata est in ommes illos qui eosdem vel aliquem seu aliquid eorundem surrepuerint seu alienaverint. vel aliquid de illo libro abraserint seu minuerint in fraudem quoquomodo. T Que omnia et singula jam collata. neenon per ipsum dominum Decanum imposterum conferenda. quilibet capellanus ejusdem cantarie pro tempore existens conservabit. reparabit et manutenebit. et amplius faciet. prout in ordinacione prefati magistri Thome Heywode plenius continetur.

Item prefatus Magister Thomas Heywode Deeanus contulit. Anno domini Millesimo. $\mathrm{CCCC}^{\mathrm{mo}}$ octogesimo primo. Altari cantarie Jesu et sancte Ame ad manus domini Johamnis Wryght capellani ejusdem cantarie. sibi et successoribus suis ibidem capellanis. ad honorem Jesu fruituris unum bonum restimentum album sacerdotale de albo veluet. cum albis paruris et singulis ipsis convenientibus. et cum nomine Jtu. sepius in paruris dicti vestimenti designato. precij. V. marcarum.

Donacio et collacio pensionim terrarum pratorum pascuarum et possessionum. Succentori et Cetui vicariorum hic nominat'. necnon servicia divina et onera que dicti vicarij supportabunt. prout est hic expressum.

Ad honorem nominis Jesu Christi. domini nostri. prefatus Magister Thomas Heywode. decanus suprascriptus devote contulit et dedit ad opus et utilitatem vicariorum choralium ecclesie cathedralis Lich. pensiones. terras. et possessiones subscriptas. videlicet quoddam messuagium in Chesturfelde. juxta Lichefelde. necnon terras. prata. pascua. et pasturas. infra parochiam de Sclienstoñ. Coventreñ et Lich. [dioc.'] situata. Ac redditum capitalem valoris annui quinque marcarum. et quinque solidorum de Johanne Teddesley. emptoz̧. IT Necnon tres pasturas vocatas Emley pyttes juxta locum qui dicitur. Hylhalmore. Et quatuor acras terre arabilis in le gaýfelde. valoris annui. xx.s. de Johanne Petý emtoz (sic). T Ac eciam novem seliones pasture. juxta ffulfengrene. divisim jacentes que vocantur. Newlande. annui valoris. ij.s. de domino Thoma 
Stykbuk. emptoz. T Necnon idem Magister. Thomas Decanus emit unum Messuagium. terras et prata apud Bromley Regis. et redditus capitales ad summam. quasi. v. s. nuper empta de Agnete relicto Thoma Pope. et nune uxor. Johamis Staunton, valoris annui. xxj. s. et ultra. et sic empta. ut prefertur. pro. xx. li. solutis dicte Agneti et domino feodi viz. Willelmo Presse pro feodo suo. xiij.s. iij d. pro introitu. xx. d. et in aliis diversis expensis. ad summam. vj. s. viij.d. Et sic summa totalis circa adepcione juris et possessionis predicte $x \mathrm{xj}$. li. que erant recepte de quinquaginta mareis deliberatis per prefatum decanum vicarijs. et positis in eorum archivis ad emendas possessiones ad valorem. xl.s. annuatim prout sequitur inferius.

Item Idem Magister Thomas Heywod emit unam parcellam prati vocatam le Brownes flat iuxta Salterbrygge in feodo de Allerwas. de Johame Browne ad valorem annum. xx. s. et pro illo fundo solvit sibi. xr. li. et pro finis et expensis ricariorum ibidem existentium in curia pro securitate illius prati. v.s. x.d. Et sic quinquaginta marce que erant posite pro securitate empcionis terrarum in baga et in cista commnni expenduntur. totaliter in ipsis duabus parcellis de Bromley. et Allerwas. et ultra de bursa dieti domini decani per quatuor marcas. et. r. s. x. d. ut patet evidenter. et sic valor dictarum duarum possessionum extendit ad $\mathrm{xlj}$. s. per annum. Annuamque pensionem quinquaginta trium solidorum et quatuor denariorum percipiendam annuatim de Abbate et conventu de Dala in comitatu Derbiensis. quam de et cum bonis sibi adeo collatis perquesivit. Et sic omnia promissa ex parte decani sunt perfecte conpleta. Deo gracias.

II Summa ommium predictorun illis collatorum. ix. li. ix. s. et plus. ut pj.

Deditque idem Magister Thomas Decanus. et eisdem vicarijs realiter deliberavit quinquaginti. marcas. ad emendas terras et tenementa de valore annuo xl. s. que summa. l. marcarum sunt. (sic) expensa pro hujusmodi empeione terrarum. ut supra scribitur. If Ac eciam ne per tardas soluciones firmarum omnium terrarum et tenementorum, annueque pensionu (sic) predicte. jacturam seu dampnum incurrerent. Valoren annui redditus omnium premissorum. viz. ix. li. x. s. dietis vicariis pre manibus contulit et donarit. If Insuper dictus Magister Thomas pro conservacione et defensione Juris dictorum vicariorum in premissis ad cistam eorum communem x. li. monete Anglie concessit. dedit pariter et liberavit. T Necnon postquam predicti vicarij ommes et singuli in loco capitulari congregati. prefatum magistrum Thomam decanum in confratrem ipsorum et participem oracionum suarum receperunt et fecerunt cum solempnitate in hujusmodi actu usitata. et in martilagio confratrem. et oracionum suarum participem scribi fecerunt. Et cuilibet vicario et adtune confratri suo libere dedit in manibus vj.s. vijj. d. ad numerum xxj. vicariorum ad tune existencium.

T Summa. vij. li.

Hinc est preterea quod Petrus Burrełt. succentor cetusque vicariorum ad tante rei gcste merioriam. pro temporalibus quantum cum Deo possunt spiritualia refundere cupientes. de roluntate et consensu expressa dominorum decani et capituli ecclesie cathedralis Licti. predict'. ac omnium singulorun aliorum quorum interest in hac parte. pia mentis intencione ex unamimi 
asscnsu omnium pariter et consensu per scripta indentata sponte et libere remotis omni dolo et fraude. pro se et successoribus suis concesserunt unam integram et solidam Cantariam perpetuam futuris temporibus in ecelesia cathedrali predicta imperpetuum duraturam. viz. unam missam apud altare in honore nominis Jesu et sancte Anne. matris beate virginis Marie. per eundem magistrum Thomam Heywode dum steterit decanus ex parte boriali ecclesie cathedralis predicte noviter in sublime erectum: per unum de convicarijs dicte ecclesie capellanum. viz. ad hoc idoneum dicendum et celebrandum omnibus diebus cujuslibet anni imperpetuum. pro bono et prospero statu ac salute corporis et anime dicti magistri Thome. dum vixerit cum oraciono et colecta speciali sub uno. Per dominum. viz. Omnipotens. sempiterne Deus. miserere famulo tuo. S.c. Ac cum secreta. Proficiat. quesumus Domine \&c. Et post communionem. Sumentes qs̃ Domine \&c. Atque similiter pro anima ejusdem Magistri Thome. cum ab hac luce migraverit in missa prefata dicetur oracio sive collecta. Deus cujus misericordie non est numerus \&c. Secreta Quesumus Domine Deus noster \&.c. Et post communionem .Presta quesumus omnipotens Deus. per hec sancta que sumpsimus \&c." Ad quam quidem missam sicut premittitur celebrandam. Succentor qui pro tempore fuerit unum vicarium cetus vicariorum ejusclem ceclesie pro tempore existencium omni ebdomada in sua tabula directoria signatum deputabit et assignabit. T Qui quidem vicarius sic deputatus et assignatus juxta exigenciam cursus sui. omni die reneris qualibet septimana sibi limitata imperpetuum missam solempnem de nomino Jesu cum nota ad altare predictum solenniter celebrabit et decantabit. seu per alium convicarium presbiterum cum collectis et oracionibus antedictis celebrari et decantari faciet. Cui quidem vicario qui missam ommi septimana modo quo prefertur celebrabit. dicti succentor et cetus vicariorum conces. serunt pro se et successoribus snis omni septimana de bonis suis communibus. xij. [d.] T Insuper prefatus succentor et cetus vicariorum concesserunt. quod in dicta missa solenni de nomine Jesu omni die veneris hora septima ut premittitur celebranda presentes erunt ante ymaginem Jesu in altari supradicto in loco congruo ad hoc desuper fabricato sex vicarij. et quatuor choriste cantatores magis idonei ecclesie cathedralis predicte per succentorem ejusdem pro tempore existentem deputandi et assignandi. qui in habitibus suis choralibus ibidem induti in missa ipsa organorum voces personari faciendo dulcius et devocius quo poterint decantabunt perpetuo in futuro. - Concedunt eciam pro se et suceessoribus suis. quod prefati sex vicarij eum quatuor choristis antedictis. per succentorem deputati. Antiphonam devotan et convenientem nomini Jesu. cum versiculis et oracionibus consuetis. ac cum prostracionibus et genufleccionibus pie devocionis affectu statim post Completorium in choro dicte ecclesie finitum. choram dicta Imagine Jesu. omni die veneris cujuscumque septimane imperpetuum. organorum melodia solenniter decantabunt. T Quibus vicarijs. quinquaginta. s. et quatuor choristis. x. s. de bonis communibus dictorum vicariorum ad quatuor anni terminos. dicti Succentor et Cetus vicariorum pro eorum laboribus et diligencijs in premissis promiserunt persolvere. Ac se et successores suos ad solucionem hujusmodi oneraverunt. et suis scriptis obligarunt. T Absente tamen vicario seu chorista

a sic.

b The writer has evidently got confused between the post communion for St. Matthias's day, and that beginning with the same words in the Salisbury mass-book in Orationes pro defunctis. 
in missa seu antiphona predicta cessabit sua solucio proporcionabiliter. prout se duxerit absentand. Nisi ille vicarius alium vicarium. et chorista. alium choristam. loco suo substituerit ad cantandum. et cantaverit in ejus absencia in premissis. T Rursus prefatus Petrus Succentor et cetus vicariorum predictorum concesserunt pro ipsis et successoribus suis. quod in Missa de Requiem per Capellanum cantarie Jesu omni die veneris ad idem altare juxta ordinacionem dicti Magistri Thome Heywode cum nota celebranda convenient iiijor vicarij ecclesie cathedralis predicte per succentorem pro tempore existentem omni die reneris qualibet septimana limitati. qui statim post missam nominis Jesu ad altare predictum eodem die celebratam. et finita dictam missam de Requiem cum nota pro animabus omnium fidelium defunctorum solenniter decantabunt. Tा Quibus quidem quatuor vicarijs. missam ipsam de Requiem decantantibus. viij. s. iiij. (l. sterlingorum ad quatuor anni terminos de bonis dictorum vicariorum communibus persolvere spoponderunt. et se et successores suos. ad solucionem hujusmodi imperpetuum oneravcrunt. et scriptis suis prenominatis obligaverunt. If Ulterius ut excitetur populus christianus ad audiendam tam missam quam antiphonam nominis Jesu predictam devocius convenire. prefati succentor et cetus vicariorum concesserunt pro ipsis et successoribus suis. quod sacrista dicte ecclesie qui pro tempore fuerit. vel ipsius quicunque minister sive familiaris. magnam campanam per spacium et intervallum competens. bis tintinnabit. et post binas tintinnaciones ante dictam missam de Jesu solemem prefatam campanam ad eandem missam congrue pulsabit. Necnon ante predicte antiphone inchoacionem bis. per intervallum competens. ipsam campanam tintinnabit seu tintinari (sic) faciet. Quodque idem sacrista omnia lumina cepi (sic) in dictis missis ct antiphona de nomine Josu necessaria et opportuna exibebit perpetuo in futurum. cui quidem sacriste pro hujusmodi luminis exibicione ac prefate campane tintinnacione ct pulsacione modo quo prefertur fiend xiij. s. iij. d. de bonis communibus [me]moratis. ad terminos supradictos fideliter solvere. promiserunt. Et idem succentor et cetus vicariorum ad solucionem hujusmodi se et successores suos forma que supra obligaverunt. I P'reterea cum Succentor ecclesie cathedralis predicte qui nume est. et inposterum erit juxta ordinacionem dicti Magistri Thome circa assignacionem et limitacionem vicariorum et chorestarum qui ut prefertur in missis de nomine Jesu. et Requiem. ac antiphonam de eoclem nomine Jesu interesse et decantare debeant multiplices labores in ea parte ac alias ut prenotatur pati debeat et teneatur. Idem succentor et cetus vicariorum volunt et pro se et successoribus suis concesserunt. quod idem succentor pro bujusmodi laboribus suis. ct ut diligenciam suam exactam adibeat in premissis. percipiat et habeat. iij.s. iiij. d. de bonis eorum communibus annuation ad quatuor anni terminos usuales sibi equis porcionibus persolvend. - Postremo prefatus succentor et cetus vicariorum pro se et successoribus suis volunt quod si quod absit premissa omnia et singula. et prout in eorum seriptis indentatis ae sigillis tam dominorum decani et Capituli et eorum communibus conscribuntur. non observaverint. seu ea in omnibus et per omnia non adimpleverint etc. bene licebit dominus Decano et capitulo ejusdem ecclesie pro tempore existentibus de bonis ipsorum vicariorum communibus rj. s. et viij. d. nomine pene exigere et levare. et in usus fabrice ecclesie cathedrali Lich. predicta convertere absque ipsorum seu successorum suorum contradiccione seu impedimento aliquali imperpetuum. T Et preter premissa ipsos et successores suos tam ad solucionem pene quam implecionem et obserracionem perpetuam omnium et singulorum premissorum sub quibuscumque sentenciis et 
censuris ecclesiasticas in eos si desides vel necligentes in premissis imposterum fuerint per decanum et capitulum dicte ecclesie pro tempore existentes. fulminandis compelli et coherceri. omnimodis appellacionibus ac juris et facti remedijs ipsis quomodolibet competentibus seu competituris postpositis omnino et semotis. If Ad que eciam omnia et singula premissa firmiter observanda. et perimplenda omni dolo et fraude postpositis et semotis. dicti succentor ct cetus vicariorum predictorum ipsos et successores suos in ipsorum puris conscientijs coram Deo et angelis Dei ejus imperpetuum et sicut volunt corrm Deo et summo judice in die extremi judicij respondere. et preter hec omnia bona sua communia presencia pariter et futura obligarunt. per scripta sua indentata. ac sigillo communi dominorum decani et capituli et communi sigillo dictorum vicariorum sigillata manifestius declarantur.

Collata vicarijs Iich' ad celebrandum (sic) missam cursalem ad altare Jesu et beate Anne sut, eorum periculo custodiend'.

Contulit eciam et de facto deliberavit idem Magister Thomas Heywod predictis vicarijs ad deserviendum altari predicto nominis Jesu et sancte Anne imperpetuum sub eorum periculis et reparacionibus in missa solemni nominis Jesu omni die veneris. et missa cursali et cotidiana per eos perpétuum celebranda ut perfertur unum bonum calicem argenteum et deauratum cum patena deaurata ponderis quasi viginti unciarum. ynameld in pede cum Imagine crucifixi. cum tribus vestimentis sacerdotalibus. unum viride de Bawdekyn pro ferialibus diebus. contulit. alterum de viride serico. cum stellis deauratis acupictum. contulit. et tercium de Wolstyd blodio. cum rubio orfrey ante et retro. et unum bonum missale magne quantitatis cum coopertorio de coreo. T Item alium librum rubrium de commemoracionibus factum et de missa Jesu. et de Requiem omni die veneris celebranda. Necnon alium parvum librum notatum de missa de Requiem omni die veneris post solennem missam de Jesu. I Item restat ibidem liber confectus de missis et cantibus. et ad honorem Jesu in missis et antiphonis Jesu omni septimana decantindis solenniter cum versiculis et collectis antiquitus devote usitatis. Et sic domino duce imperpetuum consequendis. Necnon idem vicarij predicta sic collata et imposterum conferenda sustinebunt reparabunt et reformabunt. et ipsa seu alia cum ipsa seu aliquid ipsorum defecerint scu defecerit exhibebunt suis propriis expensis imperpetuum ad predictos usus ad honorem Jesu. $\Lambda$ mell. Expense facte in fabricando novain cameram cum capella sub et supra port.tin introntus
in mansionem vicariorum.

Item prefatus magister Thomas Heywod. Decanus antedietus in prospera vita sua videlicet anno domini Millesimo $\mathrm{CCCC}^{\circ}$ septuagesimo quarto. et etatis sue sexagesimo quinto expensis suis. et de sua liberalitate vera. fabricari fecit novam cameram cum canella et alijs domibus ad lee necessarijs cum domibus inferioribus videlicet sub et supra simul cum porta introitus ibidem

VOI. LII.

$4 \mathrm{~N}$ 
similiter conjunctis videlicet ad finem venelle que ducit a villa usque ad esclesiam cathedralem Lich. ad partem occidentalem ecclesie satis decore et comodifore edificat' videlicet ad communes usus vicariorum similiterque cameram et le drawth pro infirmis vicarijs qui ad ecclesiam accedere non valent. Capella vero. ut idem infirmus cum servientibus ibidem missam audire valeat tempore congruo. Et ut in eadem capella vicarij poterunt in libris per eosdem et alios comitive collatis et ibidem positis temporibus congruis studere vel orare. Necnon ad supramedia domus satis fortis juxta cameram fabricatur et facta est pro evidencijs rotulis. thesauris suis et jocalibus ibidem corservandis. et alie domicule in eadem ad usus communes maxime comodiferos. Alie vero domus inferiores ad usus communes. ad eorum beneplacitum magis comodiferos. Qui sumptus et expense pro premissis et eorum apparatu in singulis ibidem positis et habitis. tam in plastura et vitriacione et singulis aliis prout sunt et apparent cxtendunt se ad summam xxxij. li. ut patet per parcellas in paupiris (sic) inde scriptis parcellatis.

Et notum sit universis. quod voluntas et ordinacio dicti Magistri Thome Heywode decani de voluntate et beneplacito succentoris et cetus vicariorum. hec est. Quod edificia sua predicta precipue ad supra edificata. nullo modo deputentur seu assignentur alicui private persone cetus vicariorum non infirmo. seu alteri. sed tamen cedant et occupentur modo et forma supradictis preterquam aliquis de familia regis regine principis seu ducis infra clausum ad tempus residentis. ad mandatum officiariorum suorum ibidem assignetur seu hospitetur ad tempus breve et hoc tamen cum magna difficultate societatis in quantum honeste fieri valeat contradictio fiat.

Item memorandum quod Idem Magister Thomas decanus proprius per longa tempora dedit Cetui vicariorum in numerata pecunea (sic; $\mathrm{xl}$.li. pro anniversario parentum suorum. et sui post mortem suam imperpetuum celebrando omni anno ut in indenturis inde confectis plenius continetur.

1478. Item idem Decanus fecit optimam reparacionem omnium domorum apud Chesturfelde. cum novis portis et hostijs et omnibus necessarijs ad hec. expensis suis. Anno Domini $\mathrm{M}^{\circ} \mathrm{CCCC}^{\circ}$. lxxviijo. ad summam iijor li. vij.s. vij.d. ut patet per billam de manu $M^{\prime}$ Thome Godsalfe officiarij.

Item alia vice fecit novam cameram super introitum ante. ${ }^{a}$ ac novum Kylne de nova secta in coquina posita cum expensis suis alijs ibidem ut patet intuentibus.

Item ante hec predicta idem magister Thomas dedit tres murras anglice Masers comitive vicariorum in superficiebus deauratas ad deserviendas eisdem valoris. liij. s. iiij. cl. et nuper unam mappam bonam de twyll pro alta et longa tabula in aula congruenter.

\section{Sanctificacio sive benediccio magne campane de Jesu.}

1477. Memorandum quod xiijo die Mensis Novembris videlicet in crastino sancti Bricij episcopi. Auno Domini Millesimo. $\mathrm{CCCC}^{0} .1 \mathrm{xx}^{0} \mathrm{vij}^{1100}$ Reverendus in Christi pater et dominus Dominus

a apud chestrefelde added in a somewhat later hand. 
Robertus Archadeñ episcopus, et suffraganeus domini Johannis divina gracia Covent et Licћ. episcopi. consecravit et sanctificarit maximam campanam. vocatam in vulgari. Jhe belle. in campanili ex parte australi ecclesie cathedralis Lich. habitam ex inclustria et donacione Magistri Thome Heyworl. decani ecclesie cathedralis predicte. ad honorem Dei et nominis Jesu. ac omnium sanctorum et ecclesie predicte decorem. presentibus ad tunc in dicta solemnitate in campanili predicto. dicto clecano et Magistro Rogero Walle Canonico residenciario. cum pluribus vicarijs et choristis dicentibus psalmos. et clecantantibus antiphonas et latanias (sic). prout ipsum servicium satis devotum requirebat. una cum dicto Reverendo patre consecratore dicente consecrationes. obsecraciones et oraciones decantantes satis et valde devotas. cum signis et crucibus et uncionibus quas pluribus vicibus super eandem campanam Jesu. sic descriptam in quinque partibus campane et unctam sacra unccione delibatam. I Et post hec cum a campanili usque in ecclesiam pervenerint. Dominus episcopus incepit Te Deum laudamus. Et totus chorus illuc veniens et obvians procedebat a fine ecclesie occidentali. bini. bini. cantando et solenniter complendo dicto episcopo subsequente in pontificalibus usque ad altare Jesu in parte. boriali situatum ex fundacione dicti decani ibidem celebravit et decantavit solenniter missam. cum melioribus cantatoribus ecclesie. Et omnibus completis. dictus dominus episcopus consecrator et dicti cantatores et coadjutores seu coadjurantes in domo dicti decani prandiderunt leti. In nomine

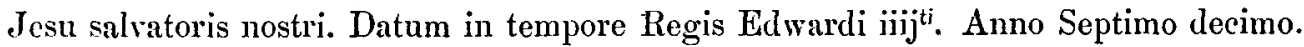

Data et collata cetui vicariorum per. M. T. Heyroode.

In primis prefatus Dominus decanus contulit cetui vicariorum. quoddam messuagium bonum in Chesturfelde juxta Lychefeld. una cum terris et pratis pasturis et pascuis ibidem annui valoris. quinque marcarum. ac redditus capitalis eidem messuagio periinentis ad summam. v.s. If Empl de Johanne Teddesley.

T Summa. V. marc'. v.s.

Item tres pasture vocate Emley pyttes juxta Hylhalmore. cum quatuor acris terre arrabilis in le Gayfecle valoris annui. xxj.s.

T Summa. xxj.s.

Item Noven seliones pasture juxta ffulfeñgrene. prout divisim jacent in campo que vocatur Newlond. valoris annui duorum solidorum. empte de domino Thoma Stykiok capellano.

ণ Summa. ij.s.

Item procuravit eisdem vicariis quamdam ammualem pensionem de abbate et conventu de Dala. in Comitatu Derbie.

T Summa. liij.s. iiij. d.

Item unum Messuagium cum terris pratis et pasturis apud Bromley regis. emptum de $4 \mathrm{~N} 2$ 
Agnete Pope. nuper uxore Thome Pope. ad valorem annualem. xxj.s. TI Item sunt eidem messuagio annexi Redditus capitales ad summam. iijor.s. et ultra.

T Summa. xxv. s.

Item unam parcellanı prati vocatam Brownesflat juxta Salters brýggo in feodo de Allerwas. emptam de Johanne Browne de eadem. et valet per annum. $x^{\text {ti }}$. s.

T Summa. $\mathrm{xx}^{\mathrm{ti}}$.s.

Summa Recepcionis omnium predictorum. ix. li, xiij.s.

Unde sex vicarij in Missa et antiphona de Jesu dêcantantes annuatim percipient. lij. s.

Item quatuor choriste decantantes in eisdem missa et antiphona percipient omni anno. x. s.

Item quatuor vicarij decantantes in missa de Requiem. omni dic veneris percipient per annum. viij.s. viij.d.

Item Succentor pro intabulacione et deputacione decantancium predictorum. percipiet ammuatin. iij.s. iiij. d.

Item Sacrista pro pulsacione et tintinacione campane de Jesu. ad predicta servicia omni die veneris per Annum percipiet. xiij s. iij. d.

Item vicarij chorales pro missa cursali per eosdem ad altare Jesu singulis diebus celebranda per annum percipient. lij.s.

T Summa solucionum pro dictis omnibus fiendis. Vj.li, xix. s. iij.'d.

Et sic omnibus et singulis persolutis pro labore et oneribus supradictis ut seriatim patet. T Adhue remanent. liij. s. viij. d. inter vicarios in communi dividenda. ad eorum commune proficium et commodum singulare eorundem.

Roopefelde. Cranefelde. et due acre terre.

4485. Et memorandum. quod prefatus Magister Thomas Decanus dedit ct concessit predictis vicarijs Anno Domini. $\mathrm{M}^{\mathrm{o}}$. $\mathrm{CCCC}^{\circ}$. $1 \mathrm{Xxx}^{0}$. $\mathrm{v}^{\mathrm{t}}$. unam pasturam vocatam Roopefylde. ad valorem de claro per ammum xiiij. s. et riij.cl. Item unum campum sive clausuram vocatam Cranefylde. precij de claro per annum. ij.s. iij. d. Item duas acras terre arrabilis in Oxonbury. precij. xvj.d. per annum. Summa omnium de claro. omnibus oneribus supportatis. xviij. s. iiij. d. que omnia emit de Johanne Palmer. de Lichfelde. et deliberavit evidencias inde dictis vicarijs. Quapropter ipsi vicarij spontanie et voluntarie concesserunt et se et successores suos obligaverunt sub sigillo suo communi. quod ipsi suis expensis exibebunt duos cereos de cera ponderis. viij. librarum. et ponentur in die parasceves omni anno ante sepulcrum immediate post imposicionem corporis 
dominici ardentes et extunc continue sic permanentes usque ad dominicam resurreccionem. Et tunc illud quod remanet et superfuerit de ipsis duobus cereis per manus sacriste vel de ejus mandato videlicet unus cereus sic remanens deliberetur capellano cantarie Jesu et beate Amne ad usus ejusdem capelle. et alius detur capellano sancti Blasij ad usus ejusdem capelle. et sic fiat annuatim imperpetuum.

On an inserted slip in the same hand:

Item prefatus dominus Decanus dedit predictis vicarijs unum pratum vocatum Simallmedoo juxta le Walle. quod emit de Thoma Halsey. quod clare valet annuatim. iij. s. iiij.d

Item edificari fecit unum bonum orreum super fundum eorum vocatum Roothall quod orreum locatur annuatim pro iij. s. vj. d.

Item deliberavit eis redditum capitalem de Chesturfeld quasi ad. v.s. Summa trium parcellarum extendit ad xxij. s. x.d. quas parcellas dedit eis ad eorum commodum in recompensum. vj. s. viij. d. quos solvit illis in supplementım de. v. marcis. et pro tenente de Chesturfeld. et exnunc omnia habent in propriis manibus. quo ad omnia onera sustinenda. ad eorum utilitatem et dicti decani exoneracionem. Et sic summa omnium parcellarum in diversis partibus conseriptarum dictis vicarijs collatarum videlicet in precedenti folio. ix.li. xiij. s. T Item supra in ista parto folij pro Ropefeld et duabus. acris in Oxenbury et Cronifeld (sic) clare xviij. s. iij. d. Et in hoc seripto trium parcellarum ad Summam. xij.s. x. d.

Unde Summa totalis omnium. xi. li. iiij.s. ij. d.

De profundis.

Item ipsi vicarij concesserunt quod in die anniversarij parentum ipsius domini Thome decani dum vixerit. et post ejus decessum iu die anniversarij parentum (sic) ipsius statim post missam de Requiem dicent unanimiter et choraliter in choro permanentes una cum canonicis residenciarijs et capellanis cantariarum novi collegij ibidem psalmum. De profundis. cum versiculis congruentibus et Oracione. Deus cui proprium est misereri. Et ad statum dicti vicarij vel unus eorum nomine dabit et ministrabit cuilibet canonico residenciario interessenti et dicenti sic cum alijs de choro. ij d. Et cuilibet vicario similiter ibidem dicenti.j.d. Et cuilibet capellano cantariarum ibidem remanenti et dicenti ut prefertur. j. d.

Item prefati vicarij vel aliquis eorum nomine dabit octo choristis dicentibus psaimum $D e$ profundis. similiter ad tunulum (sic) predicti decani et parentum suorum. statim post missam de Requiem. quamcicius simul poterunt ibidem interesse. et sic dicere pro anima dicti domini Thome decani parentum suorum. et ommium fidelium defunctorum. iiij. d. Ut in literis inde confectis et eorum sigillo communi sigillatis. plenius continetur. 
Due demonstraciones.

1481. Item in festo saneti Clementis. anno Domini. Mo. C.C.C.C ${ }^{\text {no }}$ octogesimo primo.prefatus magister Thomas Heywode. decanus in loco capitulari. coram suis confratribus. dedit ecclesic cathedrali Lich. duas capsellas. anglice monstrance argenteas deauratas et curiose fabricatas pro reliquis in eis conservandis. Et tradite fuerunt ad tunc domino Willelmo Hukyns. capellano cantarie sancti Blasij. ac custodi eapitis sancti Cedde. ad valorem. iiij. li. et xiiij. s.

Magna organa. in pulpito.

1482. Item cito post festum nativitatis Suncti Johannis Baptiste. anno Domini M․C.C.C.C ${ }^{\circ}$ octogesimo secundo. ex providencie et sumptibus magistri Thome Heywode decani antedicti. conferuntur ecclesie cathedrali Lich. organa nova magne quantitatis. et forme decentis. ad honorem sancti Cedde et ornatum ecclesie. precij. xxvi. li. iij. s. iiij. d. totaliter de sumptibus et expensis predicti decani.

If Summa. xxrj. li. iij. s. iij. d.

Organa de Jesu.

Item proprius predictus Decanus dedit unum novum par organorum ecclesie predicte. et stant in nova fabrica ex opposito altari Jesu. ad deserviend. in missa solemni de Jesu omni die reneris in sempiternum. precij xij. li.

\section{Mappa data summo altari.}

1482.

Item xij ${ }^{\mathrm{mo}}$ die mensis Marcij. Anno Domini. $\mathrm{M}^{\circ}$. $\mathrm{CCCC}^{\mathrm{mo}}$. octogesimo secundo. prefatus magister Thomas decanus sepedictus. in sua propria persona. contulit Deo. sancte Cedde. et summo altari ecelesie cathedralis Lick. suam optimam mappam de panno bilicino anglice twýly. ad usum summi altaris. et deliberatum manibus Johamnis Paxson sacriste ejusdem ecclesie. et per ipsum receptom.

Mappa data altari Jesu et sancte Anne.

Item eodem die et anno contulit altari cantarie Jesu Christi salvatoris et sancte Anne matris Marie unam mappam bonam de panno bilicino predicto. ad usum ibidem serviencium. et tradita fuit manibus domini Johannis Wrygth ad tme ejusdem eantarie capelfani perpetui. 


\section{Arappa duta altari sancti Blasij.}

- Item eodem die et anno prefatus Decanus contulit altari sancti Blasij in dicta ecclesia cathedrali Lich. ad usum perpetuum deserviencium ibidem dum duravit unam mappam bonam de twỳly. et deliberavit eam manibus domini Willelmi Hukyns. eisdem cantarie capellani.

\section{Mappa data altari uli restat caput sancti Cedde.}

Item prefatus Decanus dedit altari saneti Cedde in capella ubi caput ejus mirifice honoratur. unam mappam bonam de panno bilicino. et tradita fuit manibus domini Willelmi Hukyns ejusdem capelle custodis.

\section{Campana Jesu.}

Item antedictus Magister Thomas Heywode Decanus ecclesie Cathedralis Lich. derlit ecclesie cathedrali Lich. maximam campanam in campanili australi. Londonijs confiatam. per dominum Robertum Domini Johannis Hals episcopi suffraganium consecrata. et campana Jesu per eundum vocatam. ad valorem in tot

c. li.

\section{De vitriacione et pictura domus capitularis.}

Item idem decanus ex sumptibus suis propriis omnes fenestras domus capitularis fecit de novo vitriari cum apostolorum ymaginibus et alijs pieturis in summitate et super muros. cum tabulatu lignen sub et supra nt decet. cum vitriacione introitus de novo ad eandem. precij. xlvj. li.

\section{De tabula ad altare sancti Blasij.}

Item prefatus decanns fieri fecit tabulam de Alablastur' scultam (sic) cum historia sancti Blasij episcopi. et fecit eam poni in cape!la sancti Blasij pone altare. ubi perpetuam fundavit cantariam.

\section{Qusmodo et quare riginti marce sunt posite in thesaurio.}

Sciant presentes et futuri quod Ego Thomas Heywode decanus ecelesie cathedralis Lich. de meis propriis bonis posui viginti marcas in auro et argento. in una baga de corrio. et posita est in quadam cistula anglice a trussyng cofur. que omnia sunt posita in sista (sic) ce et cum quatuor 
clavibus in domo superiori Thesaurarie videlicet xviijo. die mensis Julij. Anno Domini. Mo.C.C.C.C. ${ }^{0}$. octogesimo tercio. ad hune finem et intencionem que secuntur (sic) Videlicet si ita contingat quod absit. quod infra decem annos primos post decessum meum ab hac luce Albas et Conventis Monasterii de Halesowen. Premonstratensis ordinis Wygornie diocesis de et super solucione novem marcarum. Seu abbas et conventus de Lyleshyll. Coventrensis et Lick. diocesis de et super solucione decem marcarum. vel Abbas sive conventus Monosterii (sic) sive domus de Dala ordinis Premonstratensis. Coventrensis et Lich. diocesis. de et super solucione annue pensionis. quatuor marcarum. Decano et capitulo ecclesie cathedralis Lich. debitarum ad usus devotos concessarum. deputatarum et ordinatarum prout in eorum literis indentatis et eorum sigillis communibns sigillatis plenius continetur. Ac per eorum superiores. et tandem per sedem apostolicam plenius in omnibus confirmatarum ut in eorum literis et bulla apostolica. sunt expressat'. in aliquo contrarium pretenderint. aut solvere contradixerint. seu terminis statutis satisfacere de facto omiserint. seu aliquis eorum contradixerit et solvere recusaverit. tune volo quod dicti contradictores et hujusmodi suas pensiones solvere temporibus congruis et assignatis recusantes implacitentur et judicialiter conveniantur. judicio ecclesiastico vel seculari prout magis convenient expedire. juxta discrecionem mandatum et supervisionem Decani et Capituli ecclesie cathedralis Lich. pro tempore existencium. de et cum expensis et pecunijs istius. et summe viginti marcarum in eadem positarum juxta juris exigenciam et racionem congruam in hujusmodi lite faciend. T Quod si nulla lis et discordia super hujusmodi solucionibus annuarum pensionum predictarum infra decem annos proximos sequentes post obitum meum habeatur seu fiat. sed ipse soluciones annue pacifice et debite persolvantur ut in predictis scriptis continetur. tune volo. et ultime voluntatis mee est. et erit in testamento meo expressatum. quod prefate viginti marce ponantur et expendantur in ornamentis ecclesie cathedralis emendis. ad Dei et saneti Cedde beneplacitum. et dicte ecclesie ornatum et decorem. juxta decani et capituli pro tempore existencium discretam et congruam voluntatem. If Et fieri volo sub obtestacione divini judicii. et majoris excommunicacionis sentencii pena. quam incurrunt ipso jure violatores et infringentes ultimam voluntatem defuncti. ipso facto. Presentibus in deliberacione hujusmodi summe pecunie. Domino Decano. R. Wall. J. Whelpedale. et T. Mille Cononicis (sic) Residenciariis. et. M. Thoma Godsalf notario publico et clerico capitulari. die mensis et anno supradictis.

\section{Acta de et super consensu Decani et Capituli de oblacionibus legatis vel alio modo collatis Imagini Jesu Christi et altari ejus ac Sancte Anne.}

Memorandum quod undecimo die mensis Aprilis. Anno Domini Millesimo. C.C.C.C. octogesimo tereio. in loco capitulari ecclesie cathedralis Lich. dominis Thoma Heywode decano. Wall. Whelpdale et Mille. Cononicis (sic) residenciarijs congregatis et Capitulum facientibus. voluerunt statuerunt et ordinarunt unanimi consensu et assensu. quod omnes oblaciones. donata. legata et col. lata Imagini Jesu Christi seu ejus altari et sancte Anne ibiderr situato ex parte borieli (sic) in ecclesia 
cathedrali predicta situato cedant et convertantur ad sustentacionem conservacionem et augmentacionem ornamentorum et paramentorum ipsius cantarie Jesu Christi ad majorem ornatum et decorem ipsius Imaginis et Capelle in qua situatur. Et post hec dicte oblaciones tonata legata et collata cedant et convertantur partim in ornamentis seu paramentis in dicta ecclesia Lich. ad divinum cultum. Ac eciam pro parte in utilitatem fabrice ccclesie cathedralis predicte.juxta piam et sinceram devocionem Decani et capituli pro tempore personaliter residencium. prout eis melius videbitur expedire. exceptis solucionibus subscriptis. integre convertantur. juxta vim forman et effectum et conservacionem perpetuam cujusdam bulle papalis et indulgensiarum domine Sixti pape quarti. Anno pontificatus sui undecimo super predietis. pro salute animarum graciose concesse et habite ut in eadem plenius continetur. Et ad majorem effectum et complecionem intencionis ipsius bulle. dicti domini decanus et capitulam voluerunt et ordinarunt quod per eosdem et suos successores omni anno eligatur et deputetur aliquis fidedignus ad predictom recipienda. et ut prefertur distribuenda. qui in inchoacione officij sui jurabit tactis sacrosanctis Dei evangelijs coram eisdem. quod erit dicte ecclesie et in officio suo diligens et fidelis. et de receptis suis et expensis ac solucionibus suis circa premissa. in fine cujuslibet anni. fidelem reddet compotum coram ipsis Decano et capitulo. It Nec grande aliquid facict cum dictis oblatis datis seu collatis sine consilio et assensu decani et capituli Licћ. pro tempore existencium vel majoris et sanioris partis eorundem. If Qui quidem officiarius sic deputatus recipiet pro labore suo et diligencia sua hujusmodi annuatim juxta arbitrium dictorum Decani et capituli racionabiliter moderatum. T Ordinarunt insuper et statuerunt dieti Decanus et capitulum quod Decanus et quilibet canonicus residenciarius qui dicto compoto prefati officiarij in loco capitulari interfuerit. et eidem diligenter et effectualiter attenderit post completum compotum percipiet per manus officiarij predicti-xij. đ. et clericus capituli presens. vj. đ. de receptis supradictis pro labore. T Residuum vero omnium oblacionum et collatorum predictorum cedant integre usibus supradictis. I Insuper prefati Decanus et capitulum. eligerunt et deputarunt eisdem die et loco dominum Johannem Wrygћt capellarum cantarie Jesu et Sancte Anne Officiarium predictum ad premissa omnia et singula facienda coram ipsis. tactis sacrosanctis evangeliis juramentum ad tune prestitit ad exequendum fideliter et diligenter ut prefertur. T Presente Magistro Thoma Godsalf Clerien capitulari. Notorio (sic) publico premissa omnia vidente et audiente. die mense et anno domini supradictis.

\section{Copia bulle papalis Indulgenciarum.}

Sixtus Episcopus. servus servorum Dei. Ad perpetuam rei memorium. Salvator noster 1481. Jesus Christus Dei filius summo patri consubstancialis et coeternus. ut humanum genus Ade primi parentis privaricacione. eterna morte dampnatum. salvum faceret. et ipsi patri reconsiliaret. de ejusdem patris sinu ad hujusmodi mundi infima descendere. et ex virgineo utero carnem nostram assumere. et tandem post habitam salutiferam inter homines conversacionem nobis que per eum ad eternam capescendam salutem datam plenam instruccionem. crucis patibulo affigi. et

VOI. LII. 40 
temporalem mortem subire dignatus est. Unde nos pie considerantes humana merita ad salutem ipsam consequendam fore penitus imparia fideles quoslibet ad visitandum ecclesias et alia pia loca spiritualibus locis muneribus indulgencijs videlicet et remissionibus libenter inducimus. ut per hoc et aliarum sanctarum operacionum exercicium. eternam beatitudinem valeant feliciter adipisci. Cupientes igitur ut. altare salvatoris domini nostri Jesu Christi et sancte Anne ad quod sicut accepimus. Dilectus filius Thomas Heywod. decanus ecclesie cathedralis Lich. unam eorundem salvatoris et sancte Anne ymaginibus in eo honorifice collocatis. et aliam ad Sancti Blasij. in eadem ecclesia sita. altaria perpetuas capellanias cantarias nuncupatas cum certo honere missarum inibi perpetuo celebrandarum aliarumque devocionum tunc expressarum suis proprijs expensis construi. et edificari fecit a Christi fidelibus congruis frequentetur honoribus. ac ut debita devocione habeatur. fidelesque ipsi eo libencius devocionis causa confluant ad eandem. et ad illius ac dicte ecclesie in qua sicut eciam accepimus ossa corporis sancti Cedde confessoris honorifice recondita existunt structurarum et edificiorum reparacionem et conservacionem. Necnon calicum librorum et aliorum ornamentorum ad divinum cultum necessariorum empcionem et fulciamentum (sic) manus provicius porrigant adjutrices. quo ex hoc ibidem dono celestis gracie uberius conspexerint se refectos. de omnipotentis Dei ac beatorum Petri et Panli apostolorum ejus auctoritate confisi. auctoritate apostolica tenore presencium statuimus et ordinamus quod omnes et singuli fideles Christi ${ }^{a}$ vere penitentes et confessi qui altare earundem Salvatoris et Sancte Anne predictum. in corporis Christi ac ac (sic) ipsius sancti Cedde. necnon sancte Anne festivitatibus. ac in die martis in ebdomada penticostes. a primis vesperis usque ad secundas vesperas eorundem festivitatum inclusive. devote visitaverint annuatim. et ad reparacionem manutencionem et fulcimentum predicta manus porrexerint adjutrices ut prefertur. septem Annos. et totidem quadragenas indulgenciarum consequantur. IT Quodque prefatus Thomas. et pro tempore existens dicte ecclesie decanus. et ipsius ecclesie dectunatu vacante presidens capituli ecclesie prefate. quatuor confessores presbiteros seculares vel cujuslibet ordinis regulares in ccclesia predicta deputare valeat. qui omnium et singulorum fidelium eorundem ad altare Salvatoris et sancte Anne hujusmodi causa indulgencie hujusmodi consequende pro tempore confluencium confessiones audire. illisque auditis. ipsos et eorum quemlibet a quibuscumque eorum peccatis criminibus delictis et excessibus dummodo taliu non sint propter que sedes apostolica esset merito consulenda. auctoritate predicta absolvere. eisquo penitenciam salutarem injungerè valeat. Presentibus perpetuis futuris temporibus duraturis. T Volumus autem quod oblaciones et pia suffragia que per ipsos Christi fideles pro hujusmodi indulgencia consequenda pro tempore fieri contigerit. in altarium [et] ${ }^{b}$ ecclesie predictorum structurarum reparacionem et [conservacionem. Necnon calicum librorum et aliorum ormamentorum hujusmodi empcione et] "

a Ipsi in MS.

b The words in brackets are omitted in the M.S. and are supplied from the recapitulation of the bulla in the succeeding document. 
fulciamentum $(\mathrm{sic})$ et non in alios usus omnino convertantur. Nulli ergo omnino homini liceat hanc paginam nostri statuti ordinacionis et voluntatis infringere vel ei ansu temerario contrarie. Si quis autem hoc attemptare presumpserit. indignacionem omnipotentis Dei ac beatorum Petri et Pauli apostolorum ejus se noverit incursurum. Dat Rome apud sanctum Petrum Anno incarnacionis dominice Millesimo C.C.C.C. octogesimo primo. pridie Idus ffebruarij. Anno pontificatus nostri Anno undecino.

Copia literarum per modum Indenture confectarum magno sigillo Capitulari sigillatarum de et super ratificacionem et collaudacionem literarum apostolicarum concessarum et indulgenciarum utlintatem " visitancium omni anno Imaginem Jesu et aliqua contribuencium. et qualiter quatuor confessores deputati. eos poterint absolvere in omnibus exceptis casubus prope reservatur. et quod omnes oblaciones collata et donata cedtant in usus predicte capelle Jesu sub forma et modo sequesitibus.

Universis Christi fidelibus presentes literas indentatas visuris et audituris. Thomas Heywode Decanus. Ac Rogerus Wall. Johannes Whelpedale. Thomas Milly. Ricardus Shirborne et Thomas Raynolde Canonici Residenciarij ecclesie cathedralis Lick. capitulariter congregati et capitulum facientes. Salutem in Domino sempiternam. Literas sanctissimi in Christo patris et domini nostri. Domini Sixti dirina providencia pape quarti. Ipsius vera bulla plumbea bullatas cum filo serico. sanas veras integras omni suspicione sinistra carentes recepimus. et diligenter inspeximus. tenorem subsequentem continentes. [Here follows the text of the papal bull as above]. T Nos vero intime considerantes. quod pia mater ecclesia de animarum salute solicita devocionem fidelium per quedam munera spiritualia remissiones videlicet et indulgencias imitare consuevit ad debitum famulatus honorem. Deo et sacris edibus impendendum ut quanto crebius et devocius illue confluit populus christianus. assiduis precibus Salvatoris graciam implorando. tanto delictorum suorum veniam et gloriam regni celestes consequi mereantur eterna. T Cupientes igitur ut ecclesia cathedralis Lich. predicta in qua confratres et canonici residenciarij vicarij et alij ministri ejusdem. ipsi Jesu Christo continue sunt famulantes. congruis honoribus frequentetur. omnia et singula in dictis literis apostolicis contenta humiliter et devote acceptamus et collaudamus. Necnon ut ea in omnibus et per omnia perpetuis temporibus oìserventur. Nos Decanus et Capitulum supradicti pro nobis et successoribus nostris volumus statnimus et ordinamus unanimi consensu et assensu nostris. quod omnes oblaciones. donata. legata et collata Imagini Jesu Christi. seu ejus alturi. et sancte Anne ibidem ex parte boriali in ecclesia cathedraii predicta situatis cedant et convertantur ad sustentacionem conservacionem et augmentacionem ornamentorum et paramentorum ipsius Cantarie Jesu Christi ad majorem ornatum et decorem ipsius Imaginis et capelle in qua

a Sic for utilitatem.

402 
situatur. Et extune residuum hujusmodi oblacionum donatorum legato:um collatorum partim in ornamontis seu paramentis in dicta ecclesia cathedrali Lich. ad divinum cultum ac eciam pro parte (et erased) ad utilitatem fabrice ecclesie Cathedralis predicte juxta piam et sinceram devocionem Decani et capituli pro tempore personaliter residencium. prout eis melius videbitur expedire. Exceptis solucionibus subscriptis integre convertatur. Et ad majorem effectum et complecionem intencionis ipsius bulle ac conservacione eiusdem. T Nos decanus et capitulum supradicti volumus statuimus et ordinamus quod aliquis fidedignus ad predictas oblaciones donata. legata. et collata recipienda. et ad usus in dicta bulla expressatos ut prefertur convertenda omni anno de cetero per nos et successores nostros eligatur et deputetur. qui in inchoacione officij jurabit tactis sacrosanetis Dei evangelijs coram nobis quod erit in officio suo circa premissa diligens et fidelis. et de receptis. expensis ac solucionibus suis per ipsum in liac parte faciendis. in fine cujuslibet anni fidelem reddet compotum coram nobis Decano et capitulo supradictis seu successoribus nostris. Nec grande aliquid faciet cum dictis oblatis. datis. seu collatis. sine consilio et assensu decani et capituli Lich. pro tempore existencium vel majoris et sanioris partis eorundem. T Qui quidem officiarius sic deputatus salarium reeipiet pro labore et diligencia sua hujusmodi annuatim juxta arbitrium dictorum decani et capituli racionabiliter moderandum. T Ordinamus insuper et statuimus nos decanus et capitnlum supradicti quod decanus et quilibet canonicus residenciarius ejusdem ecclesie cathedralis qui dicto compoto prefati officiarij in loco capitulari annuatim faciendo personaliter interfuerit. et eidem diligenter et effectualiter attenderit. post completnm compotum percipiet per manus officiarij predicti. xijd. et clericus Capituli presens. vjd. de receptis supradictis pro labore. In quorum omnium et singulorum testimonium atque fidem. Nos Decanus et Capitulum ecclesie Cathedralis Lich. antedicte presentibus Sigillum nostrum commune apponi fecimus. Data Lichefelđa. in domo nostro Capitulari xijo. die Mensis Marcij. Anno Domini Millesimo. C.C.C.C ${ }^{\circ}$. octogesimo quarto.

Donacio Magistri Thome Heywode decani Lich' facta Decano et Capitulo Lich' de certis Messuagijs. terris. pratis. pascuis. et pasturis in dominijs de Allerwas et Bromley Regis jacentibus prout sequitur.

Item memorandum quod Magister Thomas Heywode Decanus sepedictus dedit contulit et in sua ultima voluntate inter alia disposuit. quod inessuagium cum suis pertincijs in Allerwas. terre. prata. pascue. pasture et redditus capitales. que emit de Nicholao Amys de Flondesacre in possessione juxta consuetudinem curie de Allerwas. ad opus Ricardi Welles. Laurencij Smyth coci. Willelmi Rokeley ffysshemonger. et Henrici Parker de Frodley. remaneant. et redditus ac proventus eorundem remaneant imperpetuum ad usum et proficium decani et canonicorum residenci(arior)um in ecclesia cathedrali Licћ. per manus Canonici residenciarij et Communarij ejusdem ecclesie in fine anni distribuenđ. juxta ratum cujuslibet residenciarii. TI Item simili modo voluit et disposuit. quod decanus et canonici supradicti habeant et percipiant omnia Messuagia redditus prata terras pascua et pasturas in dominio de Allerwas que emit de Roberto ffysscher et juxta consue- 
tudinem curie ibidem ad opus domini Willelmi Hukyns capellani. Ricardi Welles. Willelmi Fraunces. et Christofori Harper. ad proficium ipsorum dominorum decani et Canonicorum residenciariorum eciam per manus dicti communarij ad recepcionem predictorum et solucionem prefatis decano et capitulo fienđ deputã̃. T Insuper prefatus magister Thomas Decanus voluit et ordinavit. quod predicti decanus et eapitulum habeant et percipiant in forma suprascripta quatuor pasturas suas quas emit de Willelmo Mogge Bocher de Lich. videlicet pasturam vocatam Hay by the brok. pasturam vocatam Nellfoder et pasturam vocatam Hethfelde. infra dominium de Bromley Regis existentes. que quidem quatuor pasture sic per ipsum empte sunt per cartas et munimenta in manibus suis una cum domino Johanne Paxson sacrista ecclesie Lich. Willelmo Rokeley ffysshemrnger de Lich. et Ricardo Welles juxta formam cartarum inde factarum ad intencionem ipsius decani. ut ipse faciant juxta disposicionem suam in premissis. ut prefertur. juxta majorem confidenciam quam in ipsis pre aliis posuit et prefixit ete. If Et omnes evidencias et scripturas tradidit et deliberavit eisdem. que ad predictam pertinent et coneernunt ad manus Magistri Thome Mylle canonici residenciarij. If Prefata vero omnia et singula dedit et concessit prefatus Magister Thomas decanus prefatis decano et capitulo ecclesie cathedrali Lich. pro tempore existentibus. ut orent pro salute anime sue. et ut supervideant efficaciner. quod omnia et singula divina servicia que ex Dei gracia fieri providit et ordinavit in ecclesia cathedrali Lich. predicta. debite et congrne exequantur et fiant juxta ordinaciones in ea parte factas. et per reverendum in Christo patrem et dominum dominum Johannem Dei gracia Coveñ. et Lich. Episcopum. ac Decanum et capitulum ecclesie Lich. debite et solempniter confirmatas. Ac demum per sanctissimum in Clristo patrem et dominum Dominum Sixtum divina providencia papam quartum ratificatas et confirmatas. videlicet ordinaciones suas factas de duabus Cantarijs suis in ecclesia Lich. fundatis. ridelicet unam in honore sancti Blasij martiris. Et aliam in honore nominis Jesu et sancte Anne matris beate Marie virginis. Ac de decantacione misse solempnis et antiphone de nomine Jesu omni die veneris per sex vicarios et quatuor choristas. Necnon misse de Requiem. cantande per quatuor vicarios statim post missam solempnem predictam. Et misse cursalis per unum vicarium ibidem perpetuo quolibet die celebrande. Ac eciam ordinaciones super solempni celebracione divinorum tempore anniversarij sui perpetuo annuatim celebrandi. Et aliorum serviciorum suorum in suis ordinacionibus nominatorum juxta corticem et forman ipsarum ordinacionum prout in eis plenius continetur. TI Insuper idem Magister Thomas concessit eciam ut ${ }^{2}$ oblaciones. legata. donata et collata Imagini seu altari de nomine Jesu et sancte Anne fundato cedant usui dicte capelle juxta concessionem summi pontificis predicti sub plumbo. quam concessionem et suam disposicionem prefati decanus et capitulum ad tune existentes ratificarunt. et sic fieri unanimiter concesserunt. ut in eorum Registro et in literis indentatis inde confectis ac sigillo magno communi sigillatis plenius continetur.

\section{Donacio Magisti Thome Heywode Decani facta pauperibus in domo pauperum in} Bacunstrete de duabus acris prati valoris annui vij. s. prout sequitur.

Item idem Magister Thomas Heywode Decanus prefatus. Anno Domini. MI0.C.C.C.C ${ }^{\circ}$. t $\mathbf{x x x} \mathbf{v}^{\circ}$. dedit et contulit domui pauperum. sive pauperibus in Domo elemosinaria in Lichefeld. in

a Premissa added in margin in another hand. 
vico vocato Bacunstrete degentibus sive existentibus. et successoribus suis. temporibus futuris et imperpetuum cluraturis. duas acras suas prati in feodo de Bromley Regis jacentes in le Myllenherñ. juxta le Seggeplek. ibidem. quas nuper emit de Ricardo Ruggeley et Agnete uxore ejus. de Longdon. que quidem due acre sunt in manibus dominorum Johannis Paxson. Willelmi Hukyns. et Roberto Collet capellanorum. et Willelmi Rokeley ffysshemonger de Lichíelde juxta formam et consuetudinem Curie de Bromley Regis. ut in copia curie inde facta et habita plenius continetur. Ut ipsi pauperes percipiant per manus predicte domus custodis. supervisoris. sive gubernatoris ad eorum proficium ct sustentacionem redditus inde provenientes imperpetuum. Et ut ipsi pauperes et sui successores orent pro anima dicti Magistri Thome Heywod. parentum suorum. et omnium fidelium defunctorum imperpetuum. Amen. 